\title{
Performance Comparison of Texture based Approach for Identification of Regions in Satellite Image
}

\author{
Neha Sharma \\ Punjabi University Regional Centre for IT \& \\ Mgmt. Mohali
}

\author{
Amandeep Verma \\ Assistant Professor in (CS) \\ Punjabi University Regional Centre for IT \& \\ Mgmt. Mohali
}

\begin{abstract}
Human vision is the most important resource of information used for object recognition and classification. Images having constant intensities can be easily represented by vision. Textures are one of the important features in computer vision as it identifies different regions of an image on the basis of texture properties. It is widely used in variety of applications. Identifying various regions in satellite image is one such application. There are numerous approaches based on texture classification that are mainly categorized as statistical, structural, model based and signal processing methods. The study involves the classification of LANDSAT ETM+ and MODIS satellite imagery datasets using texture based approaches i.e. Grey Level Co-occurrence Matrices (GLCM), Laws Energy Measure, Discrete Fourier Transform (DFT) and Gabor Filter. Relative performance comparison study of these approaches on the basis of standard deviation (statistical tool) has been carried out. GLCM shows best results among all other approaches.
\end{abstract}

\section{Keywords}

Texture, Satellite imagery, GLCM, Laws Energy Measure, Gabor Filter, Discrete Fourier Transform, LANDSAT ETM+, MODIS

\section{INTRODUCTION}

Satellite imagery classification can be viewed as a joint endeavor of both image processing and classification techniques. The techniques are generally used in order to assemble groups of identical pixels into classes that match the informational categories of user interest by comparing pixels to one another found in image. Image classification has emerged as a significant tool for investigating digital images.

Large quantity of satellite imagery with advanced classification technologies provides chance for useful results. Numerous classifiers have been defined. Sometimes, it is difficult to state which classifier is best for all situation as the characteristics of each image and the circumstances for each study vary so greatly. Though statistical methods based on texture approach are superior to all other classifiers but there are no clear consensuses which statistical methods work best [2]. Comparative studies of classifiers that relate their performances to data characteristics have received a lot of attention recently [14].

The paper is organized as follows: In section 2, literature is reviewed, summarizing the work presented by numerous researchers over the last three decades. Section 3 discusses the methodology and explains all four approaches i.e. Gray Level Co-occurrence matrix, Laws' Energy Measure, Twodimensional Fourier Transform and Gabor Filter that have been implemented. In section 4, k-mean clustering (classification technique) has been discussed. Results have been discussed in section 5. Conclusions and future work has been stated in section 6 .

\section{LITERATURE REVIEW}

Texture is represented on the surface of an object as a repeating pattern. It is a feature used to partition images into regions of interest and classify those regions [2]. Textures are complex visual patterns composed of entities, or subpatterns that have characteristic brightness, color, shape, size, etc. Thus texture can be regarded as a similarity grouping in an image [3]. Texture analysis methods have been utilized in many varieties of application domains ranging from classifications of satellite images [20] to biomedical images [19], detecting defects in textile products [6] to document processing [1], forensic and many more.

The vague definition of texture leads to a variety of different ways to analyze texture. They are mainly categorized as statistical, structural, model based and signal processing methods. Statistical methods [10] analyze the spatial distribution of gray values, by computing local features at each point in the image, and deriving a set of statistics from the distributions of the local features. A large number of statistical texture features have been proposed, ranging from first order statistics to higher order statistics. Structural approach [11] defines a grammar for the way that the pattern of the texture produces structure. The texture is parsed to see if it matches the grammar. The parse tree for a pattern in a particular region is used as a descriptor. Model based approaches [15] are capable of capturing dependencies among neighboring pixel values in textured images by assigning an analytical or mathematical function. Signal based or filter based approaches [21] largely share a common characteristic, which is applying filter banks on the image and compute the energy of the filter responses. It indicates texture as 2D digital signal, which are very popular and capable of dealing with random as well as regular textures. Its approaches cover a wide range of spatial and domain filtering transform, discrete transform domain analysis, and multi-scale/multidirectional methods.

\section{TEXTURE APPROACHES AND METHODOLOGY}

In this section, four different texture based approaches: Grey Level Co-occurrence Matrices, Laws Energy Measure, Discrete Fourier Transform and Gabor Filter has been discussed briefly, used for texture analysis and feature extraction.

\subsection{Grey Level Co-occurrence Matrices}

GLCM is also known as Harlick's features [18]. GLCM is a spatial dependence matrix of relative frequencies in which two neighboring pixels $i$ and $j$ have certain grey tones. Few 
parameters are considered for implementing GLCM i.e. displacement vector $d$, defined by its radius $\delta$ and orientation or angle $\theta$ [4] [5]. Co-occurrence matrices are symmetrical matrix, and its elements are expressed by:

$$
P_{i, j}=\frac{V_{i, j}}{\sum_{i, j=0}^{N-1} V_{i, j}}
$$

where $N$ represents the total number of grey levels. Haralick gave fourteen texture measures on the basis of this matrix, among which contrast, mean, variance, energy and ASM have been selected and used in this study

\subsection{Laws Texture Energy Measure}

Laws [13] presented his novel texture energy approach to texture analysis and used basic filters such as Gaussian, Edge detector and Laplacian type filters to highlight points of high 'texture energy' in the image to characterize textures highly efficiently and in a manner compatible with pipelined hardware implementations. Laws' textures are computed by first applying small convolution kernels to a digital image, and then performing a nonlinear windowing operation. A bank of separable filters [8], five in each dimension $(1 \times 5)$ i.e. a total of 25 filters were used which has been obtained by convolving three vectors of $(1 \times 3)$ dimensions i.e. Local averaging $\mathrm{L} 3=[1,2,1]$, Edge detection $\mathrm{E} 3=[-1,0,1]$ and Spot detection $\mathrm{S} 3=[-1,2,-1]$ with themselves or with each other corresponding- Level (L5), Edge (E5), Spot (S5), Wave (W5) and Ripple (R5):

$$
\begin{gathered}
L 5=L 3 \times L 3=\left[\begin{array}{lllll}
1 & 4 & 6 & 4 & 1
\end{array}\right] \\
E 5=L 3 \times E 3=\left[\begin{array}{lllll}
-1 & -2 & 0 & 2 & 1
\end{array}\right] \\
S 5=L 3 \times S 3=\left[\begin{array}{lllll}
-1 & 0 & 2 & 0 & -1
\end{array}\right] \\
W 5=-E 3 \times S 3=\left[\begin{array}{lllll}
-1 & 2 & 0 & -2 & 1
\end{array}\right] \\
R 5=S 3 \times S 3=\left[\begin{array}{lllll}
1 & -4 & 6 & -4 & 1
\end{array}\right]
\end{gathered}
$$

Once the local edginess or other feature is extracted, local magnitudes are deduced on these quantities. These magnitudes are then smoothened with a filter greater than the basic mask filter size [8]. The image gets transformed to a vector image, representing each component as energy of different type.

\subsection{Discrete Fourier Transform (DFT)}

The Two-dimensional Discrete Fourier Transform (2 DFT)

[16] of a continuous image $f(\mathrm{x}, \mathrm{y})$ is represented as:

$$
F(u, v)=\int_{-\infty}^{\infty} \int_{-\infty}^{\infty} f(x, y) e^{-2 \pi i(u x+v y)} d x d y
$$

and 2DFT for a digital image $f(\mathrm{x}, \mathrm{y})$ of size $\mathrm{M} \times \mathrm{N}$ is given by:

$$
F(u, v)=\sum_{x=0}^{M-1} \sum_{y=0}^{N-1} f(x, y) e^{-2 \pi i\left(\frac{u x}{M}+\frac{v y}{N}\right)}
$$

Generally, Image is preprocessed by applying histogram equalization. Image is transformed using Fourier transform. Complex-valued $\mathrm{F}$ is multiplied, element by element; with symmetric complex-valued filter function G. Inverse Fourier transform is calculated on filtered image to transform it back to spatial domain [16] [17].

\subsection{Gabor Filter}

It is used for edge detection in image processing, which is named after Dennis Gabor in 1946 and have emerged one of the most commonly used techniques in the field of texture analysis. These filters are based on multichannel filtering, which emulates some characteristics of the human visual system. A two-dimensional Gabor function [1] consists of a sinusoidal plane wave of some frequency and orientation, modulated by a two-dimensional Gaussian envelope. A 'canonical' Gabor filter in the spatial domain is given by:

$$
h(x, y)=\exp \left\{-\frac{1}{2}\left[\frac{x^{2}}{\sigma_{x}^{2}}+\frac{y^{2}}{\sigma_{y}^{2}}\right]\right\} \cos \left(2 \pi \mu_{0} x+\theta\right)
$$

where $\mu_{0}$ and $\theta$ are the frequency and phase of the sinusoidal plane wave along the $\mathrm{x}$-axis, and $\sigma_{\mathrm{x}}$ and $\sigma_{\mathrm{y}}$ are the space constants of Gaussian envelope along $\mathrm{x}$ - axis and $\mathrm{y}$ - axis respectively. In the spatial-frequency domain, the Gabor filter becomes two shifted Gaussians at the location of the modulating frequency [1]. The equation of the 2-D frequency response of the filter is given by:

$$
\begin{aligned}
H(u, v)=\mathrm{A}(\exp & \left\{-\frac{1}{2}\left[\frac{\left(u-u_{0}\right)^{2}}{\sigma_{u}^{2}}+\frac{v^{2}}{\sigma_{v}^{2}}\right]\right\} \\
+ & \left.\exp \left\{-\frac{1}{2}\left[\frac{\left(u+u_{0}\right)^{2}}{\sigma_{u}^{2}}+\frac{v^{2}}{\sigma_{v}^{2}}\right]\right\}\right)
\end{aligned}
$$

where $\sigma_{u}=1 / 2 \pi \sigma_{\mathrm{x}}, \sigma_{v}=1 / 2 \pi \sigma_{\mathrm{y}}$ and $\mathrm{A}=2 \pi \sigma_{\mathrm{x}} \sigma_{\mathrm{y}}$ with $\theta=0^{\circ}$ Few parameters are considered before applying Gabor filter:

Gabor Filter Banks: Frequency (F), standard deviation $(\sigma)$ and orientation $(\theta)$ are the parameters that define Gabor filter. By varying these parameters, a filter bank is obtained that covers the frequency domain almost completely.

Extraction of feature: Once the filter bank is designed, texture features can be extracted by applying various methods [7]. Among which, magnitude response was calculated on the convolved image by applying amplitude. Spatial smoothing was applied on threshold image to enhance the image followed by Gaussian post filtering in order to remove variations that occurs in magnitude image due to sinusoidal factor in Gabor filter.

\section{CLASSIFICATION TECHNIQUE: $k$ - MEANS CLUSTERING}

Once the features are extracted by using feature extraction technique, then the classification technique is applied in order to obtain clustered image. Different clustering techniques have been used for satellite image classification such as kMeans algorithm, ISODATA, Fuzzy C-Means (FCM), ISOCLUS algorithm, GAPS clustering algorithm, agglomerative clustering algorithm. In this paper k-Means clustering technique has been applied. MacQueen [12] is the pioneer of $k$-Means clustering technique. It is a simplest and most commonly used algorithm as it is easy to implement, and its time complexity is $O(\mathrm{n})$, where $n$ is the number of patterns. It starts with a random initial partition and keeps reassigning the patterns to clusters based on the similarity between the pattern and the cluster centers until a convergence criterion is met. It includes following step [4]:

i. k- Clusters are chosen and centroid coordinates are determined.

ii. Distance of each pixel to the centroid is determined.

iii. Pixels are grouped on the basis of minimum distance i.e. pixels in the image whose feature vectors represent points that are close together in the feature space. 
iv. Iteration is done until no changes result to the labels of any of the pixels.

\section{TESTS AND RESULTS}

This section tabulates the performance of every approach. Results on each technique have been discussed individually. Relative performance comparison of all approaches on the basis of standard deviation has been discussed on two different datasets.

\subsection{Datasets}

Two satellite imaging datasets LANDSAT ETM+ and MODIS have been used for classifications.

\subsubsection{LANDSAT ETM+ dataset}

LANDSAT imagery is available since 1972 from six satellites in LANDSAT series with three primary sensors: MSS (Multispectral Scanner), TM (Thematic Mapper), and ETM+ (Enhanced Thematic Mapper Plus). LANDSAT supplies high resolution visible and infrared imagery (multispectral), with thermal imagery having 8 bands that vary in resolution from 15 to 120 meters. This satellite imagery has been major component of NASA's earth observation program. The satellite images used in this study are grayscale images and are acquired from LANDSAT 7 ETM+, which is the latest in a series of LANDSAT earth observation satellites. It is used for many applications like land cover mapping, land use mapping, soil mapping, geological mapping, sea surface temperature mapping. Landsat-7 was launched in 1999 carrying the ETM+ scanner [9].

\subsubsection{MODIS dataset}

The MODIS (Moderate Resolution Imaging Spectroradiometer) sensor operates on both 'Terra' and 'Aqua' satellites. Terra's orbit around the earth is timed as it passes from north to south across the equator in the morning, where as Aqua passes from south to north over the equator in the afternoon. This study includes the classification on grayscale Terra images that are composite of sixteen days worth of images. The composites have a benefit of eliminating most cloud cover found in the images. The MODIS images are processed by NASA to calculate a number of water quality parameters [9].

\subsection{Selection of methodological parameters}

There are several methodological parameters that should be optimized as discussed. Here the results have been discussed on the basis of parameters selected, method by method. One of the most relevant parameters is the neighbourhood size. Therefore, a specific analysis is required for each of the images with a different resolution. All the input images and output images are grayscale and of size $255 \times 255$. Figure 1 (a) and Figure 1(b) shows comparative results of all texture based approaches on two different LANDSAT ETM+ dataset whereas Figure 2(a) and Figure 2(b) shows results on two different MODIS dataset.

\section{GLCM}

Window size along with the spatial displacement $(1,0)$ i.e. radius $(\delta=1)$ and angle of orientation $(\theta=0)$ has been decided. Different window sizes were applied i.e. $3 \times 3,5 \times 5,7 \times 7$, $11 \times 11,19 \times 19$. GLCM computation involves the trade-off between the window size and time, i.e. more is the window size less time is required for calculations. The increase in window size gives better results but up to some extent because when the size increases, the distance between the two neighboring pixels (i.e. reference pixel and its immediate pixel) also increases, decreasing the effect on innermost pixel. Storing GLCM requires more memory space as the total size of resultant image is $256 \times 256 \times 255 \times 255$, where $255 \times 255$ is the size of input image with $256 \times 256$ grey levels. GLCM shows good results on satellite images.

\section{Laws Texture Measure}

Convolving $5 \times 5$ laws mask or vectors to produce 25 feature images takes lots of time (less than GLCM approach) and consumes lots of memory space in order to store them. Therefore, time complexity and memory usage is very high. 1D mask have been used. For normalizing these images, any filter can be used. Here, non linear filter has been used by applying window size of $15 \times 15$. A problem in Laws energy is that the window size is bigger than the convolved mask. Though features get extracted but, it does not fit in the output window. Hence, proper clusters cannot be defined. Feature extraction results of all masks have uniform background except L5L5 mask as it does not perform on zero means. Therefore, it is suitable for distinguishing areas in the images with uniform grey level.

\section{Gabor Filter}

Multi Gabor filters has been applied with 18 banks of filters. Various set of frequencies and orientation values have been tried i.e. frequency set $\mathrm{F}=\{0.3536,0.1768,0.0884\}$ and orientation value $\theta=\{0,30,60,90,120,150\}$ that showed satisfactory results. Different values for $\sigma$ have been tested. Hit and trail method was applied which showed the best result for $\sigma \approx 4$ as compared to other values. Different values of threshold have been applied on filtered images. $0 \%$ showed no threshold. 30\%, 45\%, 70\% showed threshold, among which $30 \%$ showed best results.

\section{Discrete Fourier Transform}

Two-Dimensional Fourier Transform consumes the maximum time (approx. 12 minutes) as compared to all other algorithms due to its complexity. In order to implement TwoDimensional Fourier Transformation, the real and imaginary part has been separated on which the 2DFT has been applied using $0^{0}$ orientation value. Median filter of $3 \times 3$ mask has been applied as it does not blur the edges by making sudden jumps on brightness

\subsection{Comparison of various approaches on datasets}

In order to have performance comparison of algorithms, standard deviation, a statistical tool has been used. It has been calculated on the results of each clustered region between the two algorithms i.e. (GLCM \& Gabor), (GLCM \& DFT), (GLCM \& Laws), (Gabor \& DFT), (Gabor \& Laws) and (DFT \& Laws). Standard deviation is given as:

$$
\sigma=\sqrt{\frac{1}{N} \sum_{i=1}^{N}\left(x_{i}-\mu\right)^{2}}
$$

where $\sigma$ is standard deviation, $x_{i}$ are observed values, $\mu$ is the mean of observed values and $\mathrm{N}$ is the total number of values. The deviation values for three clustered LANDSAT ETM+ images in Table 1 has been graphical represented in Figure 3 and Figure 4 in order to show relative performance 


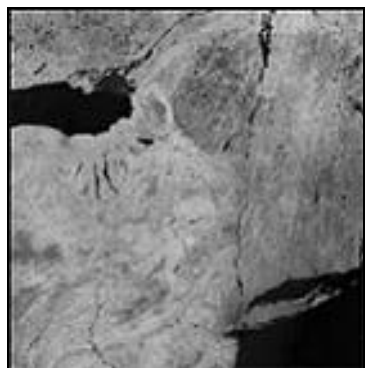

Original LANDSAT Image

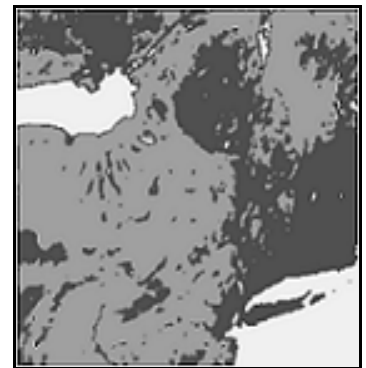

GLCM method

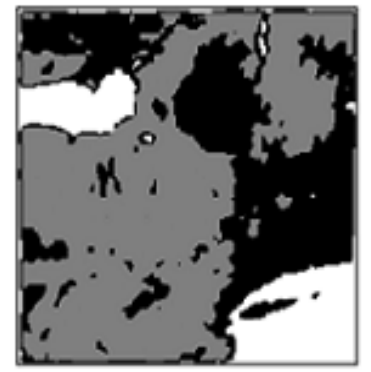

Laws Energy method

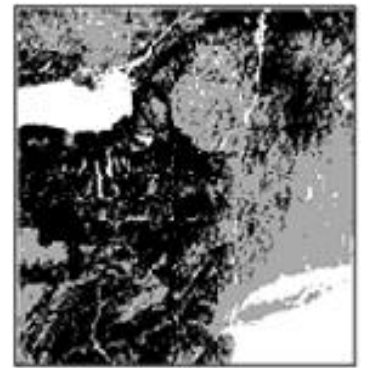

Gabor Filter method

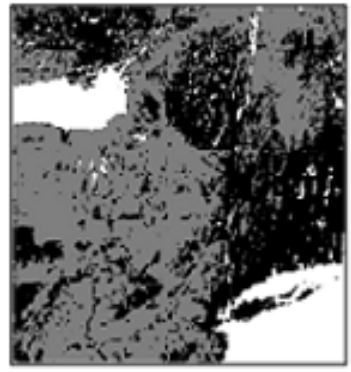

DFT method

Fig 1(a): Comparison outputs of three clustered images for all texture approaches on first LANDSAT ETM+ dataset

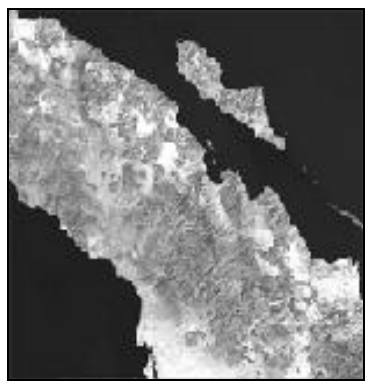

Original LANDSAT Image

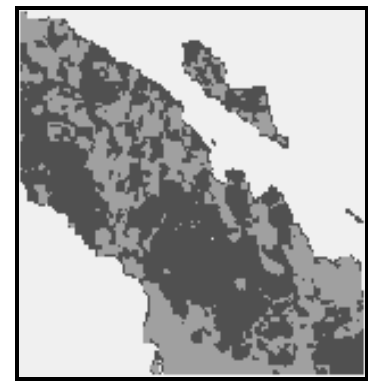

GLCM method

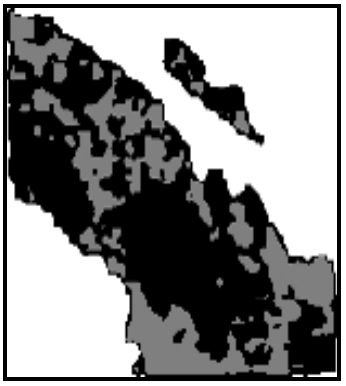

Laws Energy method

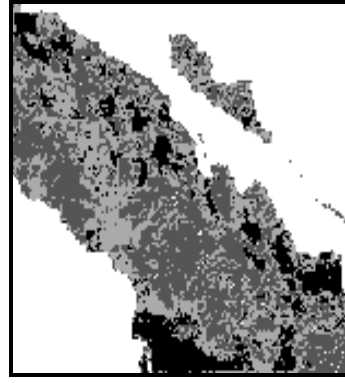

Gabor Filter method

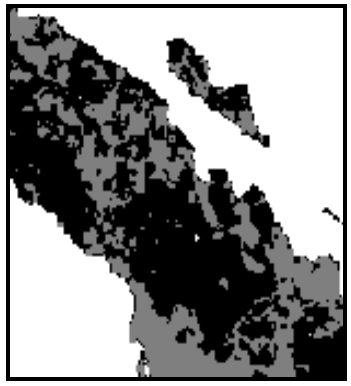

DFT method

Fig 1(b): Comparison outputs of three clustered images for all texture approaches on second LANDSAT ETM+ dataset

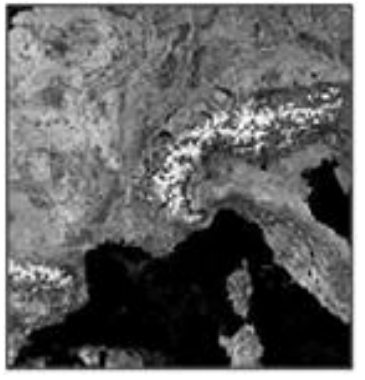

Original MODIS Image

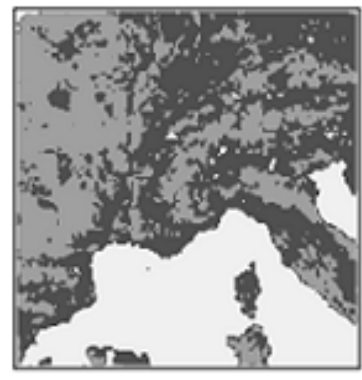

GLCM method

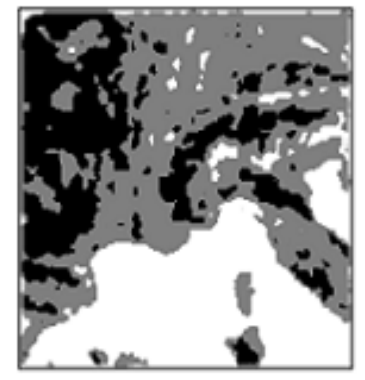

Laws Energy method

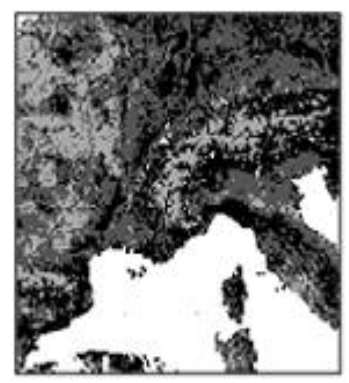

Gabor Filter method

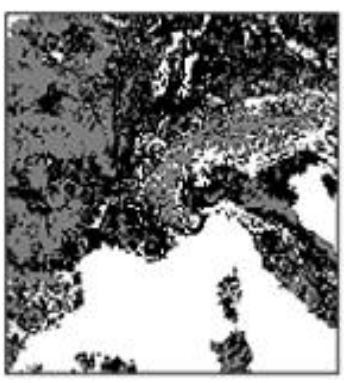

DFT method

Fig 2(a): Comparison outputs of three clustered images for all texture approaches on first MODIS dataset

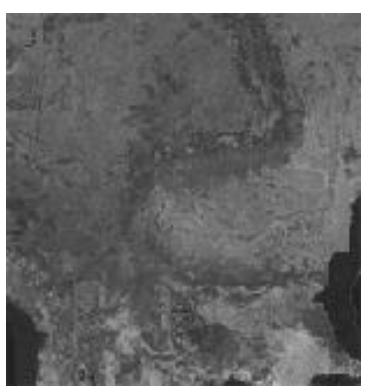

Original MODIS Image

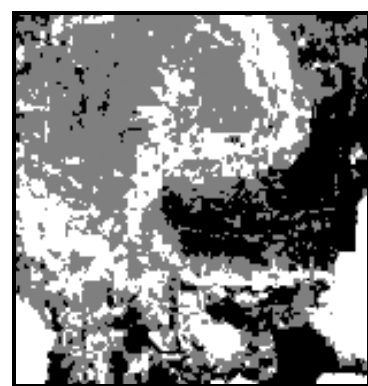

GLCM method

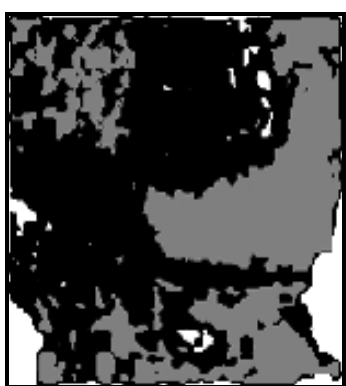

Laws Energy method

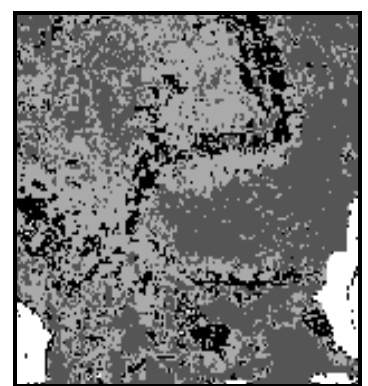

Gabor Filter method

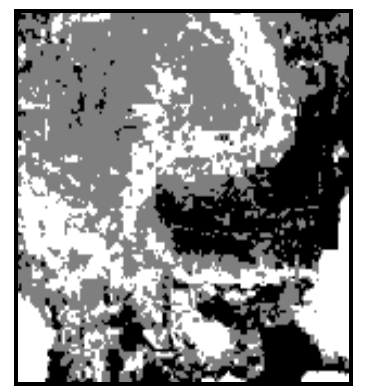

DFT method

Fig 2(b): Comparison outputs of three clustered images for all texture approaches on second MODIS dataset 
Table 1. Comparing deviation values between various algorithms for three clustered LANDSAT ETM+ images

\begin{tabular}{|c|c|c|c|c|c|c|c|}
\hline \multirow{2}{*}{ DATASET } & Clusters & $\begin{array}{c}\text { GLCM \& } \\
\text { Gabor }\end{array}$ & $\begin{array}{c}\text { GLCM \& } \\
\text { DFT }\end{array}$ & $\begin{array}{c}\text { GLCM \& } \\
\text { Laws }\end{array}$ & $\begin{array}{c}\text { Gabor \& } \\
\text { DFT }\end{array}$ & $\begin{array}{c}\text { Gabor \& } \\
\text { Laws }\end{array}$ & $\begin{array}{c}\text { DFT \& } \\
\text { Laws }\end{array}$ \\
\hline \multirow{3}{*}{ LANDSAT 1 } & Cluster 1 & 2.9818 & 6.87266 & 1.087147 & 3.892059 & 4.068742 & 7.958897 \\
\cline { 2 - 8 } & Cluster 2 & 4.418232 & 0.484253 & 0.484253 & 2.880804 & 4.902362 & 7.78183 \\
\cline { 2 - 9 } & Cluster 3 & 3.301833 & 0.848097 & 0.848097 & 1.245175 & 2.454016 & 1.208989 \\
\hline \multirow{3}{*}{ LANDSAT 2 } & Cluster 1 & 2.24948 & 6.597839 & 1.62833 & 4.349683 & 3.877506 & 8.224321 \\
\cline { 2 - 9 } & Cluster 2 & 0.373053 & 5.427073 & 1.325529 & 5.054208 & 1.698568 & 6.751719 \\
\cline { 2 - 8 } & Cluster 3 & 0.517305 & 0.7538 & 0.128873 & 0.763518 & 1.646173 & 0.882672 \\
\hline
\end{tabular}

Table 2. Comparing deviation values between various algorithms for three clustered MODIS images

\begin{tabular}{|c|c|c|c|c|c|c|c|}
\hline \multirow{2}{*}{ DATASET } & Clusters & $\begin{array}{c}\text { GLCM \& } \\
\text { Gabor }\end{array}$ & $\begin{array}{c}\text { GLCM \& } \\
\text { DFT }\end{array}$ & $\begin{array}{c}\text { GLCM \& } \\
\text { Laws }\end{array}$ & $\begin{array}{c}\text { Gabor \& } \\
\text { DFT }\end{array}$ & $\begin{array}{c}\text { Gabor \& } \\
\text { Laws }\end{array}$ & $\begin{array}{c}\text { DFT \& } \\
\text { Laws }\end{array}$ \\
\hline \multirow{3}{*}{ MODIS 1 } & Cluster 1 & 7.04375 & 9.68005 & 6.572222 & 3.639314 & 0.471772 & 4.11101 \\
\cline { 2 - 8 } & Cluster 2 & 8.50293 & 13.92253 & 10.43666 & 11.47211 & 0.935595 & 10.539 \\
\cline { 2 - 8 } & Cluster 3 & 3.393552 & 12.09082 & 5.734307 & 9.7176 & 2.342676 & 8.382962 \\
\hline \multirow{3}{*}{ MODIS 2 } & Cluster 1 & 3.252234 & 9.41267 & 0.234425 & 6.339583 & 12.34945 & 3.322805 \\
\cline { 2 - 8 } & Cluster 2 & 11.65895 & 13.02222 & 0.974459 & 10.42245 & 14.75391 & 1.273717 \\
\cline { 2 - 8 } & Cluster 3 & 6.837171 & 10.77392 & 0.487284 & 2.580815 & 2.436621 & 3.770128 \\
\hline
\end{tabular}

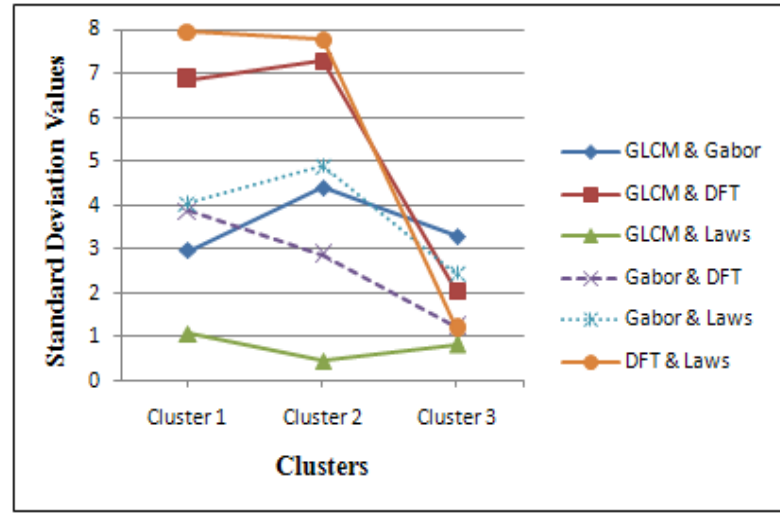

Fig 3: Relative performance comparison among various approaches for LANDSAT 1 dataset

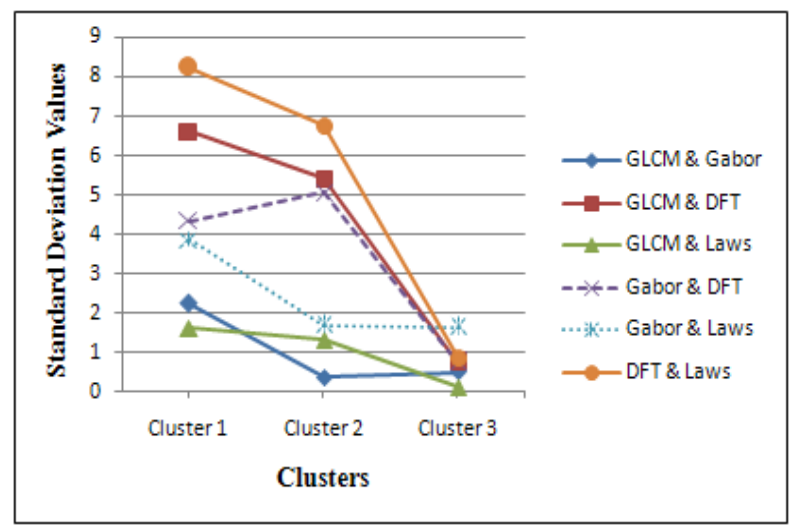

Fig 4: Relative performance comparison among various approaches for LANDSAT 2 dataset

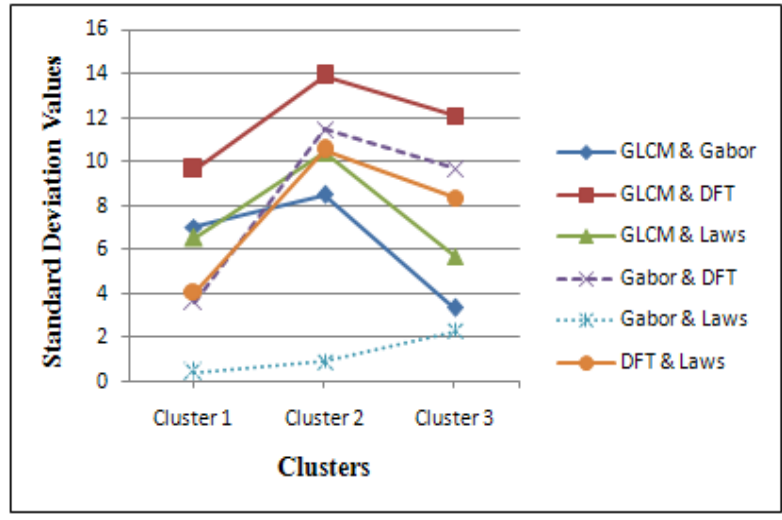

Fig 5: Relative performance comparison among various approaches for MODIS 1 dataset

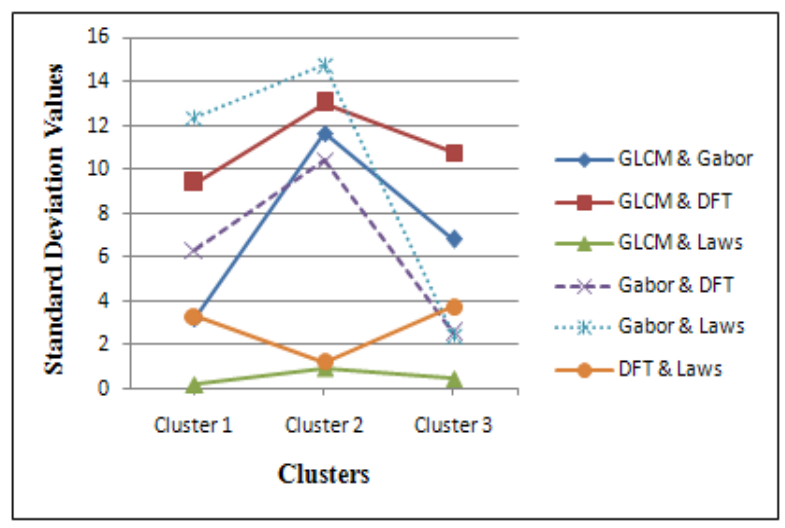

Fig 6: Relative performance comparison among various approaches for MODIS 2 dataset 
comparison among various approaches. Graphs in Figure 5 and Figure 6 illustrate the same for three clustered MODIS images as tabulated in Table 2. It is difficult to say which technique is outstanding for all the images, as all the algorithms have different characteristics like, Laws texture approach is good at edge detecting whereas GLCM is good for calculating statistical measures. Fourier transform is defined both on Gabor filter and DFT but with a difference that Gabor filter can define image both in spatial and frequency domain where as DFT can define the image only in frequency domain. According to the statistics, GLCM and Laws measure performs closely as they show small deviation results whereas both shows huge differences in the performance with DFT. Gabor Filter shows high variations with GLCM, average results with Laws measure and close results with DFT. GLCM shows the best result among all other algorithms for both LANDSAT ETM+ and MODIS by showing constant variation for all the regions.

\section{CONCLUSION AND FUTURE SCOPE}

Comparative study of various texture based algorithms for analyzing images led to satisfactory results for identifying three clustered regions on satellite images. GLCM shows the best result among all other algorithms for both LANDSAT ETM+ and MODIS by showing constant variation for all the regions. Comparing the two datasets i.e. LANDSAT ETM+ and MODIS, LANDSAT ETM+ shows better results for all the algorithms as the variations shown by algorithms in all the three clustered regions have consistency than that of MODIS. In future, this work can be extended by applying more approaches or by combining different classifiers on satellite images in order to compare them and have better results in term of less storage space and execution time. More classification regions like mountains, rocks, rivers can be identified. Other filtering techniques can be applied for preprocessing and post processing. Instead of using k-means clustering, other classification approaches can be tested on satellite images.

\section{REFERENCES}

[1] A. K. Jain and F. Farrokhnia, "Unsupervised texture segmentation using Gabor filters," Journal Pattern Recognition, vol. 24, no. 12, pp. 1167-1186, 1991

[2] A. N. Mazher and Dr. A. H. Ali, "Texture Analysis of Brodatz Images Using Statistical Methods", Engineering \& Technical Journal, vol. 29, No.4, 2011

[3] A. Rosenfeld and A. C. Kak, Digital Picture Processing, $2^{\text {nd }}$ Edition, Academic Press, Inc., Orlando, Florida, 1982

[4] A. Verma, "Identification of Land and Water Regions in a Satellite Image: A Texture Based Approach", International Journal of Computer Science Engineering and Technology, vol. 1, pp. 361-365, August 2011

[5] B. Hall, "GLCM tutorial", http://www.fp.ucalgary.ca/mhallbey/tutorial.htm,2007

[6] C. H. Chan, H. Liu, T. Kwan and G. Pang, "Automated technology for fabric inspection system," Conference on applications of automation science and technology, City University of Hong Kong, pp. 24-26, November 1998

[7] D. Clausi, M. Ed Jernigan, "Designing Gabor filters for optimal texture separability", Pattern Recognition, vol. 33, pp. 1835-1849, 2000
[8] E. R. Davies, "Introduction to Texture Analysis", In: Handbook of Texture Analysis, Mirmehdi et.al. [Editors], Word Scientific Publications, 2008

[9] F. L. Hellweger, P. Schlosser, U. Lall and J. K. Weissel, "Use of satellite imagery for water quality studies in New York Harbor", Estuarine Coastal and Shelf Science, vol. 61, No.3, pp. 437-448, 2004

[10] G.N. Srinivasan and G. Shobha, "Statistical Texture Analysis", Proceedings of World Academy of Science, Engineering and Technology, vol 36, pp. 1264-1269, 2008.

[11] J. Keng and K. S. Fu, "Syntactic Algorithms For Image Segmentation And A Special Computer Architecture For Image Processing", Doctoral Dissertation, School of Electrical Engineering, Purdue University, West Lafayette, Indiana, Dec 1977

[12] J. B. MacQueen, "Some methods for classification and analysis of multivariate observations", In Proceedings of the Fifth Berkeley Symposium on Mathematical Statistics and Probability, Berkeley, University of California Press, pp. 281-297, 1967

[13] K. I. Laws, "Texture energy measures", In Proceedings of the Image Understanding Workshop, pp. 47-51, November 1979

[14] L. A. Ruiz, A. F. Sarría and J. A. Recio, "Texture feature extraction for classification of remote sensing data using wavelet decomposition: A comparative study", International Archives of the Photogrammetry, Remote Sensing and Spatial Information Sciences, vol. 35, Part B4, pp. 1109-1114, 2004

[15] M. Tuceryan and A. K. Jain, "Texture Analysis", In: The Handbook of Pattern Recognition and Computer Vision, C. H. Chen, L. F. Pau, P. S. P. Wang [Editors], $\left(2^{\text {nd }}\right.$ Edition), World Scientific Publishing Company, pp.207248, 1998

[16] R. Gonzalez, C. Woods and E. Richard, Digital Image Processing, (3 ${ }^{\text {rd }}$ Edition), Addison-Wesley Longman Publishing Co., Inc., Boston 1992

[17] R. Klette, K. Schluns and A. Koschan, "Computer Vision: Three-Dimensional Data from Images", Springer Singapore, 1998

[18] R. M. Haralick, K. Shanmugam and Dinstein, "Texture features for image classification”, IEEE Transactions on Systems, Man and Cybernetics, vol. SMC-3, No.6, pp. 610-622, November 1973

[19] R. S. Sabeenian and V. Palanisamy, "Texture-based medical image classification of computed tomography images using MRCSF", International Journal of Medical Engineering and Information, vol. 1, Issue 4, pp. 459472, 2009

[20] S. Charaniya, T. Patwardhan, A. Verma, "Texture Based Image Analysis", CSCI 8820- Computer Vision and Pattern Reorganization, Term Paper, 2008

[21] V. S. Vyas and P. Rege, "Automated Texture Analysis with Gabor filter", GVIP Journal, vol. 6, Issue 1, pp. 3541, 2006 